\title{
Orthopaedic/Radiology/Pathology Conference
}

\author{
A Slow-growing Anterior Tibial Mass in a 37-year-old Woman
}

\author{
Scott O'Neal MD, Benjamin K. Potter MD, \\ Sheila C. Adams MD, J. David Pitcher Jr. MD
}

Published online: 19 May 2009

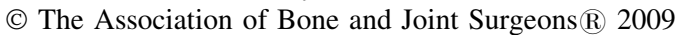

\section{History and Physical Examination}

A 37-year-old Haitian woman who was 6 months' pregnant presented with a 6-year history of a slowly enlarging mass on her anterior lower left leg. The patient stated the mass had been present for many years with recent growth during the last 6 years. The patient had been pregnant five times and she reported the mass had been stable in size between pregnancies with episodic growth during the pregnancies. She denied any history of trauma, night pain, fevers, chills, or other constitutional symptoms. She also denied any functional pain with activity.

Physical examination revealed a 6-cm nontender mass located on the anteromedial aspect of her left lower leg (Fig. 1). The mass was firm on palpation, immobile, and appeared adherent to the underlying tibia. The overlying skin displayed no erythema, cutaneous changes, or draining sinuses. There was a nearly imperceptible scar located at the

Each author certifies that he or she has no commercial associations (eg, consultancies, stock ownership, equity interest, patent/licensing arrangements, etc) that might pose a conflict of interest in connection with the submitted article.

Each author certifies that his or her institution approved or waived approval for the reporting of this case and that all investigations were conducted in conformity with ethical principles of research.

This work was performed at University of Miami/Jackson Memorial Hospital.

\section{S. O’Neal ( $₫)$, S. C. Adams, J. D. Pitcher Jr.}

Department of Orthopaedics, University of Miami/Jackson

Memorial Hospital, PO Box 016960 (D-27), Miami, FL 33101,

USA

e-mail: soneal@med.miami.edu

B. K. Potter

Walter Reed Army Medical Center Orthopaedic Surgery

Service, Washington, DC, USA distal aspect of the mass; however, the patient specifically denied any knowledge of an injury to her leg. The patient had no palpable lymphadenopathy in the groin region, and there were no other similar masses noted throughout her body.

Imaging studies including plain radiographs (Fig. 2) and contrast-enhanced MRI (Fig. 3) were performed.

Based on the patient's history, physical examination, and imaging studies, what is the differential diagnosis?

\section{Imaging Interpretation}

Radiographs revealed a soft tissue lesion located on the anteromedial aspect of the tibia with elevation and thickening of the cortex and periosteum. There was scalloping at the interface of the mass and the tibia (Fig. 2), but no invasion of the medullary canal. It was unclear whether the lesion was cortically based with secondary extension into the soft tissues or centered in the soft tissues with associated extrinsic erosion of the bone. No calcifications or mineralization was evident in the mass. MRI revealed a heterogeneous, well-circumscribed, juxtacortical subcutaneous mass (Fig. 3). T1-weighted axial and sagittal images revealed relatively decreased signal intensity versus skeletal muscle. T2-weighted sequences showed more heterogeneity, with some areas isointense to skeletal muscle and other areas hyperintense compared with skeletal muscle. The lesion also showed heterogeneous central enhancement after gadolinium contrast administration.

\section{Differential Diagnosis}

Juxtacortical versus intracortical hemangioma

Periosteal osteosarcoma 


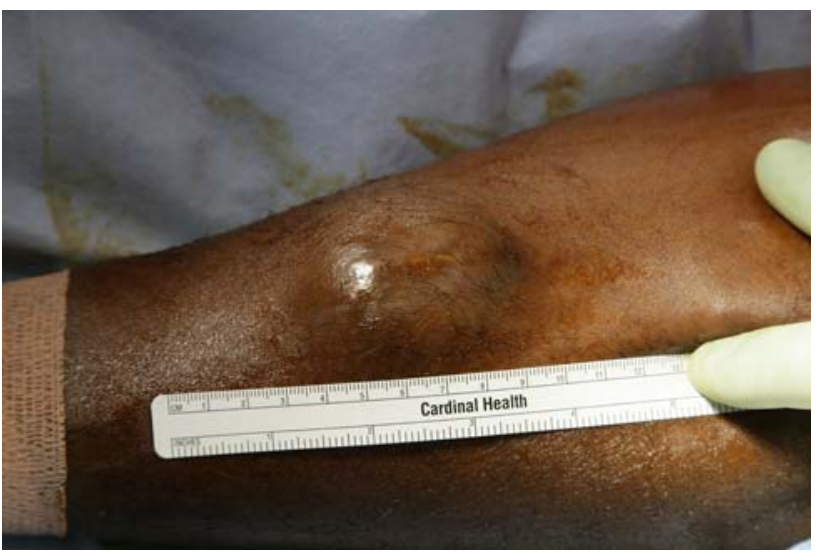

Fig. 1 An intraoperative clinical photograph of the patient's anteromedial left leg shows an approximately $6-\times 4-\mathrm{cm}$ soft tissue subcutaneous mass. No overt skin breakdown or draining sinuses were present, but a small scar is noticeable over the central aspect of the mass.

\section{Chondrosarcoma}

Atypical infection

Soft tissue sarcoma (with secondary osseous changes)

A core needle biopsy was performed in the office, and the core sample was sent for histopathologic analysis (Fig. 4).

Based on the history, physical examination, imaging studies, and histologic appearance, what is the diagnosis and how should this lesion be treated?

\section{Histology Interpretation}

Histopathologic examination of the surgical specimen and core needle biopsy showed septated and branched hyaline hyphae (Fig. 4). The hyphae were interspersed between connective tissue and there was a histiocytic reaction present. The hyphae had a dark pigmentation and were organized into nodules.

\section{Diagnosis}

Atypical infection, specifically, fungal mycetoma.

\section{Discussion and Treatment}

A 37-year-old woman presented with a 6-year history of a slow-growing mass on the anterior aspect of her left leg. Based on the lesion's prolonged benign growth pattern, its location on the patient's lower extremity, and most importantly, the histologic findings from the core needle biopsy, a diagnosis of fungal mycetoma was made. The differential included juxtacortical versus intracortical hemangioma, atypical infection, periosteal osteosarcoma, chondrosarcoma, and soft tissue sarcoma with secondary osseous changes.

Based on the radiographic findings and the periods of growth during pregnancy, we initially placed hemangioma at the top of our differential diagnosis. Reactive cortical thickening or hyperostosis has been reported secondary to hemangiomas [11], and the MRI showed image intensity and enhancement patterns that could be interpreted as flow voids. Some hemangiomas have hormone receptors and respond to estrogen stimulation, and we surmised the reported periods of growth only during pregnancy were a response to the elevated levels of estrogen $[3,10]$. However, after gross and histologic examination of the core needle biopsy, our presumptive diagnosis was changed to
Fig. 2A-B (A) Anteroposterior and (B) lateral radiographs of the patient's left leg show diffuse thickening of the tibial cortex underlying the soft tissue mass with a focal area of scalloping or erosion into the cortical bone.
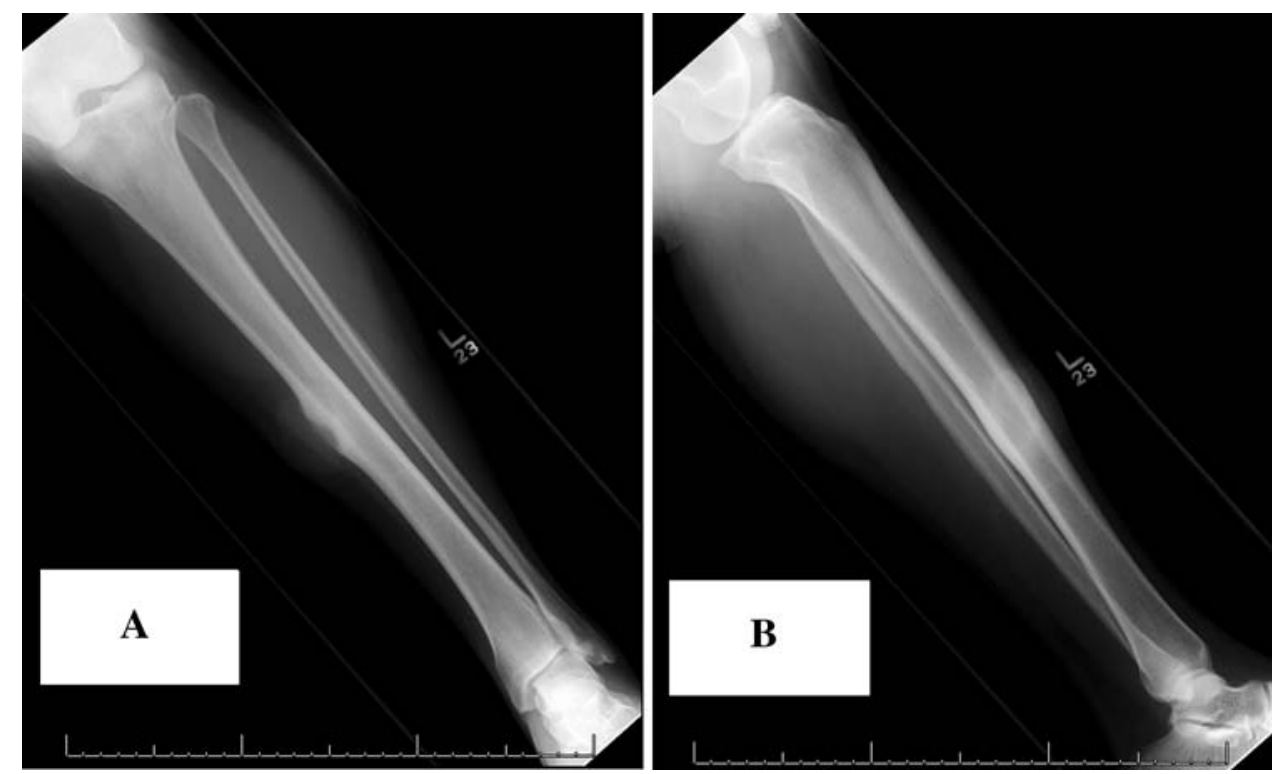
Fig. 3A-D (A) Axial T1-weighted, (B) sagittal T1-weighted, (C) axial T2-weighted, and (D) axial T1-weighted, fat-suppressed, gadoliniumenhanced MR images show a juxtacortical subcutaneous soft tissue mass of the anteromedial leg with mild heterogeneity and central contrast enhancement.
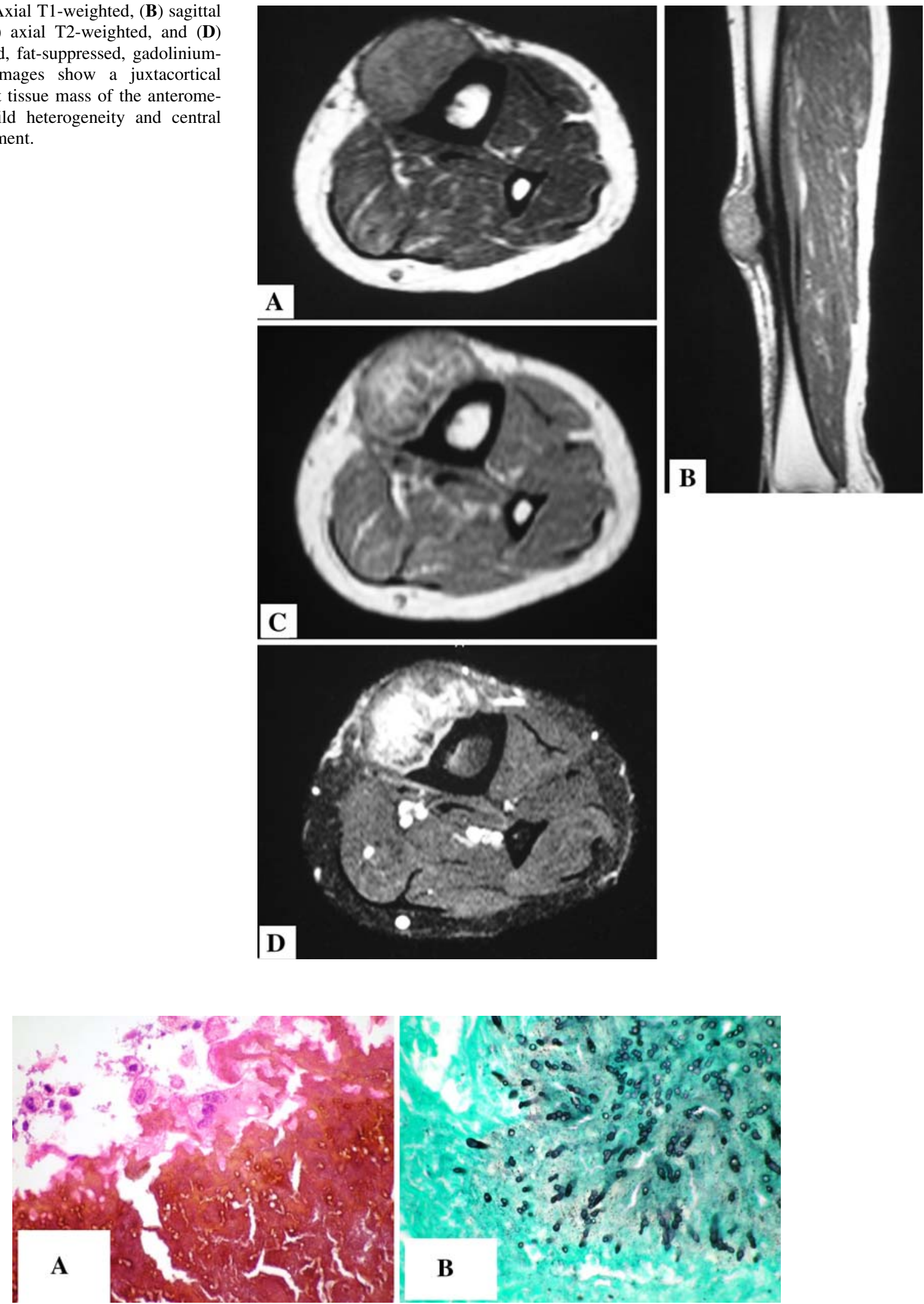

Fig. 4A-B Photomicrographs of (A) the core biopsy specimen and (B) the surgically resected mass show fungal hyphae in connective tissue with a histiocytic reaction. The hyphae have a dark brown pigmentation, which suggests the organism is a dematiaceous fungus. Focally viable fungi are further characterized by septated and branched hyaline hyphae. The fungal organisms are arranged in nodules exhibiting black pigmentation. 
atypical infection, in this particular case, a fungal mycetoma. Periosteal osteosarcoma is a rare surface form of osteosarcoma, which typically occurs on the diaphysis of long bones and rarely invades the medullary canal. The radiographic appearance of this lesion typically would show mineralization in the juxtacortical mass, which was not seen on this patient's preliminary radiographs. Chondrosarcoma is typically an intramedullary lesion with secondary extension into the soft tissues after cortical breakthrough. In addition, with a chondrosarcoma, one would expect radiographic signs of cartilage matrix production: arcs, rings, flocculations, and stippled calcifications. In our case, initial radiographs of the lesion did not show calcifications and the lesion appeared to be based outside the medullary canal. Soft tissue sarcoma with secondary osseous changes was a consideration based on the radiographs and MRI, but given the long duration of symptoms, this diagnosis was considered lower on the differential. After the results of the core needle biopsy, this diagnosis was rejected owing to the absence of malignancy and the presence of fungal hyphae.

Mycetoma is a rarely reported disease process in the United States and therefore is considered an atypical infection in this region. This entity, however, continues to be a substantial problem in African countries such as Senegal, Sudan, Kenya, Chad, North Cameroon, Somalia, and Niger [4]. Mycetoma is a chronic, progressive, inflammatory, granulomatous infection of subcutaneous tissue, skin, and bones. Characteristic findings are subcutaneous swelling and the presence of the etiologic agent contained in granules on histologic examination [7]. The infecting agent may spread contiguously to involve adjoining skin with the formation of multiple sinuses. It may be caused by true fungi (eumycetes) or by higher filamentous bacteria (actinomycetes); therefore, it is classified into eumycetoma and actinomycetoma, respectively [2]. It follows a penetrating injury, which inoculates soil organisms into the subcutaneous tissue. This particular infection occurs preferentially in rural areas, usually among laborers who work barefoot [6].

Our patient's presentation is quite common for the development of a mycetoma. The inoculating event usually is not noticed by the patient and the early part of the disease is typically benign and characterized by painless focal swelling. If pain is present, it is usually secondary to a superimposed bacterial infection. The dorsal foot and lower leg are the two most common sites of inoculation. The initial host response is a typical neutrophilic reaction followed by formation of a granuloma [1]. As the infection progresses, the disease typically spreads along fascial planes, with hematogenous or lymphatic spread being uncommon. The painless subcutaneous nodules eventually will indurate and rupture, forming a sinus tract [9]. The infection then will spread along the contiguous fascial planes forming new sinus tracts as old tracts close down. This leads to considerable disfigurement and functional morbidity to the affected body part as the infection continues to progress. The natural course of the disease is measured in years when left untreated [1]; however, cases of actinomycetes tend to progress more rapidly [8].

The initial evaluation begins with a basic history and physical examination, including a detailed travel history specifically involving tropical or subtropical areas. In this case, our patient spent most of her life in Haiti before immigrating to the United States. The next step in the evaluation includes laboratory and radiographic studies. Basic infection laboratory tests, including a complete blood count, C-reactive protein, and erythrocyte sedimentation rate, also should be obtained. In this particular case, owing to the patient's concurrent pregnancy, we believed laboratory tests would not be useful for infectious markers. Radiographic studies, MRI, and ultrasound may provide useful information, with ultrasound particularly valuable in the evaluation of a vascular tumor. We chose not to order an ultrasound, as the patient initially presented to our office with good-quality MRI and radiographs.

It is critical to differentiate between an actinomycetoma, caused by aerobic bacteria, and a eumycetoma, caused by true fungi, as the treatment is markedly different. This critical distinction can be determined with histologic inspection, cytology, culture, and immunostaining [8]. Ketoconazole or itraconazole combined with aggressive surgical débridement is recommended for patients with eumycetoma. An actinomycetoma typically will respond to antibacterial agents alone in the early portion of the disease, and different therapeutic options can be used depending on the causative organism and its sensitivities [5].

Given the patient's long and indolent clinical course and her impending delivery, treatment was delayed until after delivery. After an uncomplicated vaginal delivery, the patient underwent an en bloc excision of the mass with saucerization of the anterior tibia and primary closure of the overlying skin (Figs. 5, 6). Based on the histopathologic analysis noted above, the mass was consistent with a eumycetic mycetoma; however, fungal cultures at the time of biopsy and surgery failed to identify a causative organism after 30 days incubation. Following the recommendations of our infection disease staff, we instituted empiric therapy with $200 \mathrm{mg}$ itraconazole by mouth twice a day for 12 to 18 months. At her 1-year followup, she has had no recurrence of the mass and no constitutional symptoms and is asymptomatic with regard to her healed operative incision. We plan continued surveillance for recurrence of the infection with serial clinical examinations and MRI at the completion of her itraconazole therapy. 
Fig. 5A-C Intraoperative photographs show (A) prominent venous vascular channels on the periphery of the mass, (B) black necrotic material at the massbone interface, and (C) healthyappearing bleeding bone after resection and saucerization of the mycetoma bed.

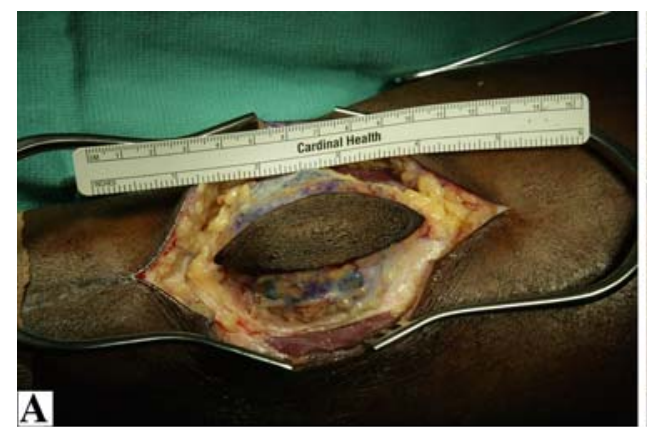

A

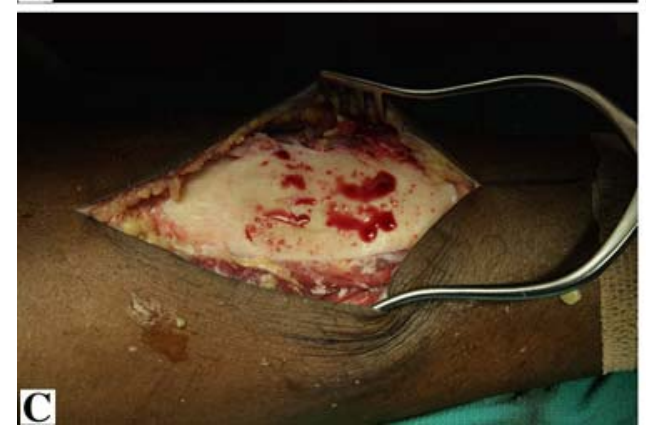

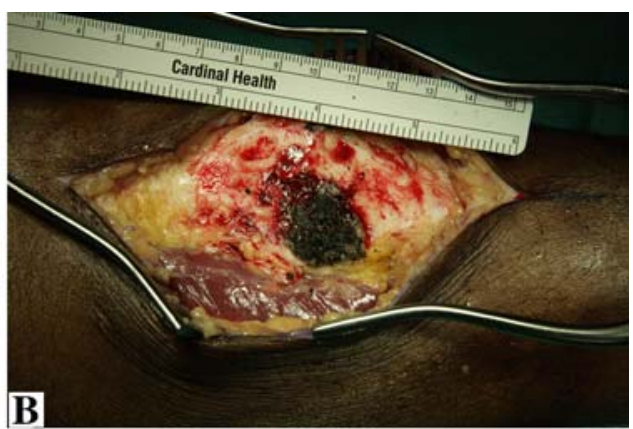

B
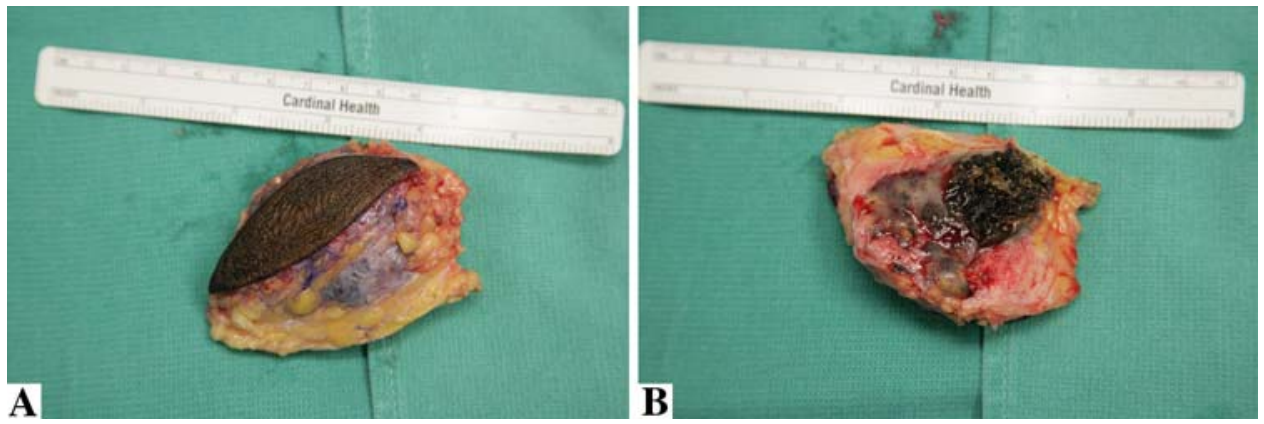

Fig. 6A-B (A) A gross pathology photograph of the resected specimen shows prominent venous channels and a scar from previous trauma. (B) The bone-mycetoma interface shows the black necrotic material and some reactive cortical bone surrounding the necrotic material.

It is important to maintain a high index of suspicion for an infectious agent causing a focal mass. With this in mind, it is essential to remember the maxim: biopsy every infection and culture every tumor.

\section{References}

1. Ania B, Asenjo M, Kiel R. Mycetoma. eMedicine from WebMD. Available at: http://emedicine.medscape.com/article/211459overview. Accessed December 2, 2008.

2. Baki R, Mathur DR. Incidence and changing pattern of Mycetoma in western Rajasthan. Indian J Pathol Microbiol. 2008;51:154-155.

3. Chatzoulis G, Kaltsas A, Daliakopoulos S, Sallam O, Maria K, Chatzoulis K, Pachiadakis I. Co-existence of a giant splenic hemangioma and multiple hepatic hemangiomas and the potential association with the use of oral contraceptives: a case report. J Med Case Reports. 2008;2:147.
4. Develoux M, Audoin J, Treguer J, Vetter JM, Warter A, Cenac A. Mycetoma in the Republic of Niger: clinical features and epidemiology. Am J Trop Med Hyg. 1988;38:386-390.

5. Develoux M, Dieng MT, Kane A, Ndiaye B. [Management of mycetoma in West-Africa] [in French]. Bull Soc Pathol Exot. 2003;96:376-382.

6. Dieng MT, Sy MH, Diop BM, Niang SO, Ndiaye B. Mycetoma: 130 cases. Ann Dermatol Venereol. 2003;130(1 pt 1):1619.

7. Fahal AH. Mycetoma: a thorn in the flesh. Trans R Soc Trop Med Hyg. 2004;98:3-11.

8. Lee DK, Schwartz AK. Primary osteomyelitis of the calcaneus with active subcutaneous nodules. J Foot Ankle Surg. 2007;46: 302-306.

9. Lichon V, Khachemoune A. Mycetoma: a review. Am J Clin Dermatol. 2006;7:315-321.

10. Scheinfeld NS. Pyogenic granuloma. Skinmed. 2008;7:37-39.

11. Sung MS, Kang HS, Lee HG. Regional bone changes in deep soft tissue hemangiomas: radiographic and MR features. Skeletal Radiol. 1998;27:205-210. 